\title{
Relapsing Bacteraemia due to Corynebacterium striatum in a Patient with Peripheral Arterial Disease
}

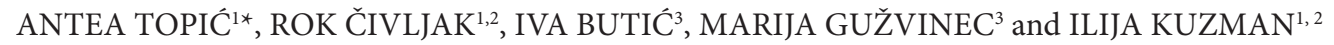 \\ ${ }^{1}$ Department of Acute Respiratory Diseases, University Hospital for Infectious Diseases “Dr. Fran Mihaljević”, \\ Zagreb, Croatia \\ ${ }^{2}$ University of Zagreb School of Medicine, Zagreb, Croatia \\ ${ }^{3}$ Department of Microbiology, University Hospital for Infectious Diseases “Dr. Fran Mihaljević, Zagreb, Croatia
}

Submitted 28 December 2014, revised 11 April 2015, accepted 13 April 2015

\begin{abstract}
We describe the first reported case of Corynebacterium striatum (C. striatum) relapsing bacteraemia in a patient with peripheral arterial disease and proven Corynebacterium species colonization of a chronic foot ulcer, focusing on the difficulties in the management of the patient. We conclude that the optimal duration of the antibiotic treatment for relapsing C. striatum bacteraemia from a chronic ulcer should be 6 weeks together with surgical treatment.
\end{abstract}

Ke y wo rds: Corynebacterium striatum, antibiotic treatment, peripheral arterial disease, relapsing bacteraemia, skin infection

Corynebacterium species other than Corynebacterium diphteriae are part of the normal flora of human skin and mucous membranes (Coyle and Lipsky, 1990). Corynebacterium striatum (C. striatum), a Gram-positive bacillus, was traditionally regarded to be a colonizer or a contaminant (Watkins et al., 1993). The first published case of C. striatum infection was in 1980 in an immunocompromised patient with pleuropulmonary lesions (Bowstead and Santiago, 1980). Since then, the frequency of $C$. striatum community acquired and nosocomial infections has increased significantly. Reported cases of $C$. striatum infections include a wide variety of different types of infections: bacteraemia (Dall et al., 1989; Martin et al., 2003; Tumbarello et al., 1994; Watkins et al., 1993), endocarditis (Marull and Casares, 2008; Mashavi et al., 2006; Fernandez Guerrero etal., 2013; Tran et al., 2012), central catheter infection (Martinez-Martinez et al., 1997; Chen et al., 2012), meningitis (Hoy et al., 1997; Weiss et al., 1996), pleuropneumonia (Cowling and Hall, 1993; MartinezMartinez et al., 1994; Renom et al., 2014; Severo et al., 2014), osteomyelitis (Fernandez-Ayala et al., 2001), arthritis (Cone et al., 1998; Scholle, 2007; Westblade et al., 2014), and intrauterine infection (Martinez-Martinez et al., 1997). Person to person transmission in intensive care units (Brandenburg et al., 1996; Iaria et al., 2007;
Leonard et al., 1994) and a number of skin infection cases like pyogenic granuloma, infected ischemic ulcer, breast abscess and a skin ulcer in an HIV patient (Bottone et al., 2010; Peiris et al., 1994; Stone et al., 1997; Watkins et al., 1993) have also been described. Although C. striatum has been linked to chronic ulcer infection and bacteraemia (Martin et al., 2003; Martinez-Martinez et al., 1997), to the best of our knowledge, no patient with relapsing bacteraemia after appropriate antibiotic treatment has been reported. We present a case of a 61-y-old man with C. striatum relapsing bacteraemia, peripheral arterial disease and proven Corynebacterium species colonization of chronic foot ulcer, focusing on the difficulties in the management of the patient.

A 61-y-old man was admitted to the University Hospital for Infectious Diseases, Zagreb, Croatia in November of 2013 because of fever and a painless ulcer of his right foot stump. He had been sick for $14 \mathrm{~d}$ with fever and oral clindamycin was prescribed, but without effect. His medical history was remarkable for coronary heart disease $(1 \mathrm{y}$ earlier the patient underwent PCA stent insertion and was treated with beta-blocker, ACE-inhibitor, statin and antiplatelet drugs) and $10 \mathrm{y}$ severe peripheral arterial disease with development of gangrene of right foot which resulted in amputation (at the level of the Chopart's joint). During the 6-months

\footnotetext{
* Corresponding author: A. Topic, Department of Acute Respiratory Diseases, University Hospital for Infectious Diseases “Dr. Fran Mihaljević", Zagreb, Croatia; e-mail: antea.topic@gmail.com
} 
prior to the admission of the patient, the ulceration formed with sporadic non-purulent secretion followed by debridement, antibiotic treatment (oral clindamycin) and hyperbaric oxygen treatment with consecutive good healing. The patient was a heavy smoker.

On admission the patient was febrile $\left(38^{\circ} \mathrm{C}\right)$, but in good general condition with blood pressure of $130 / 80 \mathrm{mmHg}$, pulse $90 / \mathrm{min}$ and respirations $18 / \mathrm{min}$. On examination a $1.5 \mathrm{~cm}$ ulcer was present above his right foot stump with no surrounding erythema, tenderness or purulent drainage. Right inguinal lymph nodes were enlarged up to $2 \mathrm{~cm}$, painless and elastic. The pulse of his dorsal artery of the foot was faint. Meningeal signs were negative, the patient had no signs of pharyngitis or oral mucosal ulcers, but his oral cavity was in poor condition. His chest and cardiac examination were unremarkable. The abdomen was soft and painless, the liver was palpable $2 \mathrm{~cm}$ below the right costal margin, while the spleen was not palpable.

Laboratory tests revealed an increased erythrocyte sedimentation rate $(70 \mathrm{~mm} / \mathrm{h})$, C-reactive protein was $141.8 \mathrm{mg} / \mathrm{l}$ and the total white blood count was $9.0 \times 10^{9} / 1$ with mature neutrophilia. The red blood cell count was $4.49 \times 10^{12} / \mathrm{l}$, haemoglobin was $131 \mathrm{~g} / \mathrm{l}$ and the platelet count $133 \times 10^{9} / 1$. The levels of sodium, potassium, chloride, urea nitrogen, creatinin, glucose, total proteins (albumin and globulin), total bilirubin, aminotransferases, lactate dehydrogenase and alkaline phosphatase were normal; only the level of gamma glutamyl transferase was increased at $184 \mathrm{IU} / \mathrm{l}$. Routine coagulation tests were normal with no signs of disseminated intravascular coagulation, but the level of fibrinogen was $8.0 \mathrm{~g} / \mathrm{l}$. Urinalysis revealed no abnormalities. The serologic HIV test was negative. Chest X-ray and electrocardiogram were normal. Abdominal ultrasound examination revealed a mild hepatomegaly and small gallstones without inflammation of the gallbladder. Radiographs of the right leg showed no findings of osteomyelitis. Both transthoracic and transesophageal echocardiograms were normal.

Gram-stained smear of scrapings of the ulcerative lesion showed a presence of Gram-positive bacilli without polymorphonuclear leucocytes and the culture revealed Corynebacterium species. Four separate sets of blood culture taken at different times on admission grew C. striatum susceptible to vancomycin, imipenem, penicillin and amoxicillin-clavulanic acid. The strain was identified using the commercial system of cultivation BacT/ALERT (bioMerieux, France) and identification system VITEK 2 (bioMerieux, France). Upon identification, the strain was confirmed by an in house method of sequence analysis of the internal fragment of the 16S rRNA gene (Savini et al., 2013), using no control, but comparing the final sequence to the one in the PubMed database. Antibiotic susceptibility was deter- mined using E-test (bioMerieux, France) for penicillin, amoxicillin-clavulanic acid, clindamycin, imipenem and vancomycin following the recommendations of the European Committee for Antimicrobial Susceptibility Testing (EUCAST). E-test values revealed that all isolates were resistant only to clindamycin. The $\mathrm{MIC}_{\mathrm{s}}$ were as follows: penicillin $0.12 \mathrm{mg} / \mathrm{l}$, amoxicillin-clavulanic acid $0.19 \mathrm{mg} / \mathrm{l}$, vancomycin $0,5 \mathrm{mg} / \mathrm{l}$, imipenem $0.032 \mathrm{mg} / \mathrm{l}$ and clindamycin $>256 \mathrm{mg} / \mathrm{l}$.

The initial antibiotic treatment with intravenous amoxicillin-clavulanic acid was continued for $15 \mathrm{~d}$ after the diagnosis was established. Defervescence occurred $1 \mathrm{~d}$ after the initiation of the treatment and surveillance cultures were negative on day 8 . Seven days after the amoxicillin-clavulanic acid treatment ended (during which the patient developed a mild Clostridium difficile diarrhoea and was treated with oral metronidazole) recurrence of the fever occurred and two sets of repeated blood culture were positive for C. striatum of identical antibiotic susceptibility. Based on the clinical presentation and positive blood culture, intravenous vancomycin was started and after clinical improvement of the patient, was continued for $15 \mathrm{~d}$. The patient fully recovered and was discharged from the hospital with instructions of taking oral amoxicillin-clavulanic acid for another 4 weeks. During these 4 weeks, the patient also underwent surgical treatment (debridement) of the ulcer, which finally resulted in the healing of the ulcer of the right foot. During a 3 month follow up no fever was noted.

C. striatum, a coryneform bacteria usually considered a contaminant, is a non-sporulating, non-acid-fast pleomorphic gram-positive rod that is aerobic and facultatively anaerobic (Coyle and Lipsky, 1990). It was until recently that $C$. diphteriae was considered to be the only pathogen of the coryneform species. Today, besides $C$. striatum, other well known pathogens of the species include Corynebacterium jeikeium, Corynebacterium urealyticum, and Corynebacterium amycolatum (Funke et al., 1997). Most cases of C. striatum infection occurred either in immunocompromised patients or patients whose skin barrier integrity was broken, rather than in previously healthy persons, because of C. striatum's low adhesive properties and low pathogenicity. Neither toxin nor other virulence factors explain the transmission from contamination to infection (Watkins et al., 1993). The number of reported cases of diseases generated by C. striatum in the past few decades, and especially in the last couple of years, has been on the rise due to the improvement of microbiological techniques and the survival of patients with underlying diseases. C. striatum bacteraemia is a rare event and is scarcely documented in literature (Chen et al., 2012; Dall et al., 1989; Martin et al., 2003; Martinez-Martinez et al., 1997; Tumbarello et al., 1994; Watkins et al., 1993). Associated 
conditions include diabetes, cirrhosis, chronic renal failure, trauma, surgery and malignancy.

The case we have described is the first reported case of a relapsing bacteraemia in a patient with a proven source of infection (in this instance a chronic foot ulcer) despite appropriate treatment. Several factors promoted the risk of C. striatum infection; the presence of a foot ulcer as a result of a compromised peripheral arterial circulation, age, and previous antibiotic treatment. As has previously described (Leonard et al., 1994), we also presume that, in our patient, selective pressure due to a prior antibiotic consumption favoured the overgrowth of C. striatum.

Corynebacteria are common skin and culture media contaminants, and discrimination between colonization and infection is, in some cases, difficult. In our case, the presence of four consecutive blood cultures positive only for. C. striatum suggests that this microorganism was the likely pathogen. Although the C. species cultivated from the foot ulcer was not identified to species level, because it was regarded as a contaminant, the skin-circulation route is most likely. Some authors (Martin et al., 2003) have demonstrated by molecular techniques (PCR) identical strains of C. striatum from the skin and the bloodstream in a patient with peripheral arterial disease, confirming the entry of the bacterium trough the skin to the circulation. In our case, as a colonizer of the patient's skin, C. striatum established a de novo bacteraemia despite antibiotic treatment. Probable causes of the relapsing bacteraemia are a (too) short antibacterial treatment, choice of antibiotics, and vasculopathy.

The optimal duration of antibiotic treatment for. C. striatum bacteraemia is not known (Fernandez-Roblas et al., 2009; Martinez-Martinez et al., 1996), but the clinical course of the infection in the presented patient (recurrence of fever and bacteraemia) suggests a need for a prolonged antibiotic treatment (we recommend 6 weeks) together with surgical treatment. If aggressive debridement is performed earlier, we presume that much shorter course of antibiotic treatment may be sufficient.

In conclusion, this case highlights the growing importance of C. striatum as a serious pathogen and the fact that consecutive positive blood cultures for corynebacteria, and C. striatum in particular, should never be overlooked.

\section{Literature}

Bottone E.J., M. Fabbri and A. Ashraf. 2010. Corynebacterium striatum: Chronic infection of a cutaneous ulcer in a patient with AIDS. Rev. Infect. 1: 104-109.

Bowstead T.T. and S.M. Santiago. 1980. Pleuropulmonary infection due to Corynebacterium striatum. Br. J. Dis. Chest. 74: 198-200.
Brandenburg A.H., A.Van Belkum, C. Van Pelt, H.A. Bruining, J.W. Mouton and H.A. Verbrugh. 1996. Patient-to-patient spread of a single strain of Corynebacterium striatum causing infections in a surgical intensive care unit. J. Clin. Microbiol. 34: 2089-2094.

Chen F.-L., P.-R. Hsueh, S.-O. Teng, T.-Y. Ou and W.-S. Lee. 2012. Corynebacterium striatum bacteremia associated with central venous catheter infection. J. Microbiol. Immunol. Infect. 45: 255-258. Cone L.A., N. Curry, M.A. Wuestoff, S.J. O'Connell and J.F. Feller. 1998. Septic synovitis and arthritis due to Corynebacterium striatum following an accidental scalpel injury. Clin. Infect. Dis. 27: 1532-1533.

Cowling P. and L. Hall. 1993. Corynebacterium striatum: a clinically significant isolate from sputum in chronic obstructive airways disease. J. Infect. 26: 335-336.

Coyle M.B. and B.A. Lipsky. 1990. Coryneform bacteria in infectious diseases: clinical and laboratory aspects. Clin. Microbiol. Rev. 3: 227-246.

Dall L., W.G. Barnes and D. Hurford. 1989. Septicaemia in a granulocytopenic patient caused by Corynebacterium striatum. Postgrad. Med. J. 65: 247-248.

Fernandez-Ayala M., D.N. Nan and M.C. Farinas. 2001. Vertebral osteomyelitis due to Corynebacterium striatum. Am. J. Med. 111: 167. Fernandez-Roblas R., H. Adames, N.Z.

Martin-De-Hijas, D.G. Almeida, I. Gadea and J. Esteban. 2009. In vitro activity of tigecycline and 10 other antimicrobials against clinical isolates of the genus Corynebacterium. Int. J. Antimicrob. Agents 33: 453-455.

Fernandez Guerrero M.L., I. Robles, C. Nogales Mdel and D. Nuevo. 2013. Corynebacterium striatum: an emerging nosocomial drug-resistant endocardial pathogen. J. Heart Valve Dis. 22: 428-430.

Funke G., A. Von Graevenitz, J.E. 3rd Clarridge and K.A. Bernard. 1997. Clinical microbiology of coryneform bacteria. Clin. Microbiol. Rev. 10: 125-159.

Hoy C.M., K. Kerr and J.H. Livingston. 1997. Cerebrospinal fluidshunt infection due to Corynebacterium striatum. Clin. Infect. Dis. 25: 1486-1487.

Iaria C., G. Stassi, G.B. Costa, C. Biondo, E. Gerace, A. Noto, S.G. Spinella, A. David and A. Cascio. 2007. Outbreak of multiresistant Corynebacterium striatum infection in an Italian general intensive care unit. J. Hosp. Infect. 67: 102-104.

Leonard R.B., D.J. Nowowiejski, J.J. Warren, D.J. Finn and M.B. Coyle. 1994. Molecular evidence of person-to-person transmission of a pigmented strain of Corynebacterium striatum in intensive care units. J. Clin. Microbiol. 32: 164-169.

Martin M.C., O. Melon, M.M. Celada, J. Alvarez, F.J. Mendez and F. Vazquez. 2003. Septicaemia due to Corynebacterium striatum: molecular confirmation of entry via the skin. J. Med. Microbiol. 52: 599-602.

Martinez-Martinez L., A. Pascual, K. Bernard and A.I. Suarez. 1996. Antimicrobial susceptibility pattern of Corynebacterium striatum. Antimicrob. Agents Chemother. 40: 2671-2672.

Martinez-Martinez L., A.I. Suarez, M.C. Ortega and R. RodriguezJimenez. 1994. Fatal pulmonary infection caused by Corynebacterium striatum. Clin. Infect. Dis. 19: 806-807.

Martinez-Martinez L., A.I. Suarez, J. Rodriguez-Bano, K. Bernard and M.A. Muniain. 1997. Clinical significance of Corynebacterium striatum isolated from human samples. Clin. Microbiol. Infect. 3: 634-639.

Marull J. and P.A. Casares. 2008. Nosocomial valve endocarditis due to Corynebacterium striatum: a case report. Cases J. 1: 388.

Mashavi M., E. Soifer, D. Harpaz and Y. Beigel. 2006. First report of prosthetic mitral valve endocarditis due to Corynebacterium striatum: Successful medical treatment. Case report and literature review. J. Infect. 52: e139-141. 
Peiris V., S. Fraser, C. Knowles, S. Norris and C. Bennett. 1994. Isolation of Corynebacterium striatum from three hospital patients. Eur. J. Clin. Microbiol. Infect. Dis. 13: 36-38.

Renom F., M. Gomila, M. Garau, M.D.C. Gallegos, D. Guerrero, J. Lalucat and J.B. Soriano. 2014. Respiratory infection by Corynebacterium striatum: epidemiological and clinical determinants. New Microbes New. Infect. 2: 106-114.

Savini V., G. Gherardi, M. Favaro, C. Fontana, R. Marrollo, A.V. Argentieri, G. Dicuonzo, P. Fazii and D. D'antonio. 2013. About a bloodstream Corynebacterium striatum isolate. Folia Microbiol. (Praha) 58: 451-453.

Scholle D. 2007. A spontaneous joint infection with Corynebacterium striatum. J. Clin. Microbiol. 45: 656-658.

Severo C.B., L.S. Guazzelli, M.B. Barra, B. Hochhegger and L.C. Severo. 2014. Multiple pulmonary nodules caused by Corynebacterium striatum in an immunocompetent patient. Rev. Inst. Med. Trop São Paulo 56: 89-91.

Stone N., P. Gillett and S. Burge. 1997. Breast abscess due to Corynebacterium striatum. Br. J. Dermatol. 137: 623-625.
Tran T.T., S. Jaijakul, C.T. Lewis, L. Diaz, D. Panesso, H.B. Kaplan, B.E. Murray, A. Wanger and C.A. Arias. 2012. Native valve endocarditis caused by Corynebacterium striatum with heterogeneous high-level daptomycin resistance: collateral damage from daptomycin therapy? Antimicrob. Agents Chemother. 56: 3461-3464.

Tumbarello M., E. Tacconelli, A. Del Forno, S. Caponera and R. Cauda. 1994. Corynebacterium striatum bacteremia in a patient with AIDS. Clin. Infect. Dis. 18: 1007-1008.

Watkins D.A., A. Chahine, R.J. Creger, M.R. Jacobs and H.M. Lazarus. 1993. Corynebacterium striatum: a diphtheroid with pathogenic potential. Clin. Infect. Dis. 17: 21-25.

Weiss K., A.C. Labbe and M. Laverdiere. 1996. Corynebacterium striatum meningitis: case report and review of an increasingly important Corynebacterium species. Clin. Infect. Dis. 23: 1246-1248.

Westblade L.F., F. Shams, S. Duong, O. Tariq, A. Bulbin, D. Klirsfeld, W. Zhen, S. Sakaria, B.A. Ford, C.-A.D. Burnham and C.C. Ginocchio. 2014. Septic arthritis of a native knee joint due to Corynebacterium striatum. J. Clin. Microbiol. 52: 1786-1788. 